\title{
Thermal neutron intensity measurement with fission chamber in current, pulse and Campbell modes
}

\author{
Shakir Zeinalov ${ }^{*}$, Olga Sidorova ${ }^{1}$, Pavel Sedyshev ${ }^{1}$, Valery Shvetsov ${ }^{1}$ \\ Youngseok Lee ${ }^{2}$, Uk-Won $\mathrm{Nam}^{3}$ \\ ${ }^{1}$ Joint Institute for Nuclear Research, 141980, Dubna Moscow region, Russia \\ ${ }^{2}$ National Fusion Research Institute, Daejon, Republic of Korea \\ ${ }^{3}$ Korea Astronomy \& Space Science Institute, Daejon, Republic of Korea
}

\begin{abstract}
In thermal nuclear reactors, most of the power is generated by thermal neutron induced fission. Therefore, fission chambers with targets that respond directly to slow neutrons are of great interest for thermal neutron flux measurements due to relatively low sensitivity to gamma radiation. However, the extreme conditions associated with experiments at very low cross section demand highly possible thermal neutron flux, leading often to substantial design changes. In this paper we report design of a fission chamber for wide range (from 10 to $10^{12} \mathrm{n} / \mathrm{cm}^{2} \mathrm{sec}$ ) measurement of thermal neutron flux. Test experiments were performed at the first beam of IBR2 pulsed reactor using digital pulse processing (DPP) technique with modern waveform digitizers (WFD). The neutron pulses detected by the fission chamber in each burst $(5 \mathrm{~Hz}$ repetition rate) of the reactor were digitized and recorded to PC memory for further on-line and off-line analysis. New method is suggested to make link between the pulse counting, the current mode and the Campbell technique.
\end{abstract}

\section{Introduction}

In recent decade digital pulse processing (DPP) is considered as very powerful alternative to traditional analogue pulse processing (APP) in experimental nuclear physics research. Mainly it happened due to a diversity options were provided in data analysis and the flexibility of data treatment, which very unlikely can be provided by APP. In this work we demonstrated the power of DPP implemented in thermal neutron counting with fission chamber (FC) in the various modes developed in the reactor instrumentation, namely the counting, the current and the Campbelling techniques. We demonstrated how to implement DPP making direct link between the counting and the current mode measurement with the thermal neutron sensor based on gas filled detectors. It should be noticed that absolute thermal neutron flux measurement in the wide intensity range was very unlikely possible with the same mode. The main problem was in the response function of the sensor, which has limit in the width about few tens of ns. When the distance between pulses became comparable with this limit, then separation of the individual pulses was impossible. In that case measurement usually was possible to perform in the current mode, using the proportionality of the measured current to the neutron intensity. The main problem is how to make link between the counting and the current modes. In this work we propose a solution which we believe able to provide the link.

\section{Experimental Setup and Results}

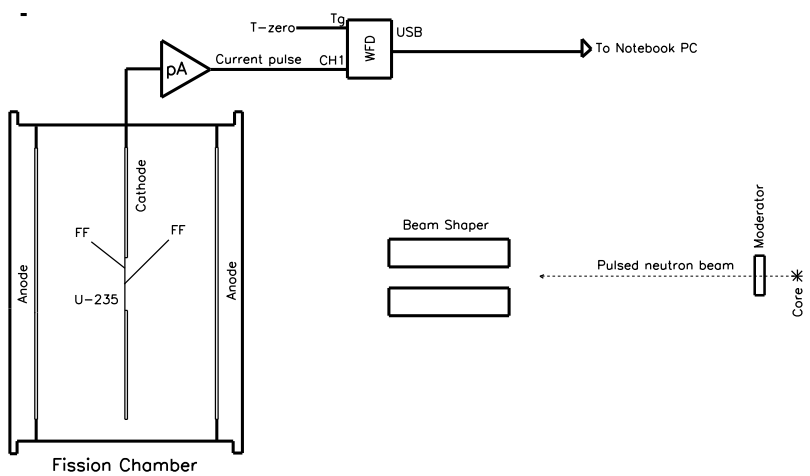

Fig. 1. Fission chamber with associated preamplifier, WFD and beam shaping equipment

In reported experiment we used the fission chamber with ${ }^{235} \mathrm{U}$ target $(99,999 \%$ enrichment $)$ mounted on the common cathode of a twin parallel plate ionization chamber shown in Fig. 1. The target thickness was 0.5 $\mathrm{mg} / \mathrm{cm}^{2}$ of uranium smeared on the both sides of aluminium backing foil. Ionization chamber was operated at constant flow $50 \mathrm{ml} / \mathrm{min}$ of working gas P10. Negative high voltage $(500 \mathrm{~V})$ was applied to the common cathode allocated between two anodes at distances of $40 \mathrm{~mm}$ from each of them. Due to thickness of the target and backing foil only one of two fission fragments (FF) was detected on thermal neutron induced fission event. Working gas pressure was stabilized at 1.05 bar using a gas pressure controller. Fission chamber was located at distance $27 \mathrm{~m}$ from the pulsed nuclear reactor IBR2 with the following main parameters: average power $1.8 \mathrm{MW}$, fast neutron pulse duration 200 us, the pulse repetition rate $5 \mathrm{~Hz}$, the thermal neutron intensity at the target position was about $10^{6} \mathrm{n} / \mathrm{cm}^{2}$ per

\footnotetext{
* Corresponding author: zeinal@nf.jinr.ru
} 
second. Fission fragments (FF) were decelerated in the working gas of FC producing free electrons which were drifted towards the anodes under the applied uniform electric field, inducing electric current pulses on the cathode circuit. The cathode current was amplified and recorded by the WFD, triggered by the IBR2 "T-zero" signal. The WFD sampling frequency $(200 \mathrm{kHz})$ and the number of samples (4096) were selected in order to cover the full thermal neutron burst duration about 20 ms. Sequential reactor bursts were digitized and recorded to the notebook computer's hard disk for further off-line data analysis. One of the recorded reactor bursts presented in Fig.2.

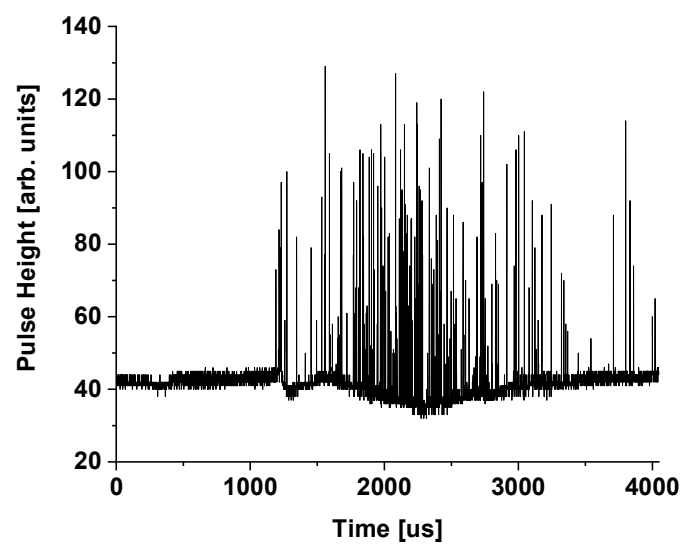

Fig. 2. Recorded waveform of single burst of the IBR-2. Full range is 20500 us

It should be noticed that because of the overlapped pulses baseline was distorted and must be restored using special electronic modules to stabilize the base line for proper counting when the APP was implemented. Use of the DPP avoided the problem in the elegant way, using sequential flow of the reactor bursts. To remove the baseline fluctuation we have used the following method: each recorded waveform of the burst was subtracted from the waveform of previous one, creating waveform shown in Fig. 3. It was easy to see that resulted distribution composed of bipolar waveform with removed baseline and the data obtained was satisfying to the second Campbell theorem [1,2]. According to the theorem the variance of the current's AC component from the randomly distributed in time (Poisson distribution) current pulses is proportional to the average pulse rate and the mean square charge per pulse:

$$
\begin{aligned}
& \langle I(t)\rangle=\int_{0}^{\infty} h(t) d t \cdot \sum_{i=0}^{m}\left\langle n_{i}\right\rangle \cdot\left\langle q_{i}\right\rangle \\
& \left\langle I(t)^{2}\right\rangle-\langle I(t)\rangle^{2}=\int_{0}^{\infty} h(t)^{2} d t \cdot \sum_{i=0}^{m}\left\langle n_{i}\right\rangle \cdot\left\langle q_{i}^{2}\right\rangle
\end{aligned}
$$

where, $h(t)$ the circuit's response to a single pulse of a unit charge, $\left\langle q_{i}\right\rangle$ the mean charge per pulse, $\left\langle q_{i}^{2}\right\rangle$ the mean square charge per pulse, $\left\langle\boldsymbol{n}_{\boldsymbol{i}}\right\rangle$ the average pulse rate in a fixed interval of measurement. In current measurement $\langle I(t)\rangle^{2}=0$, because the current waveform $I(t)$ was residue of two sequential waveforms. So the Eq. 2 reduced to Eq. 3:

$$
\left\langle I(t)^{2}\right\rangle=\int_{0}^{\infty} h(t)^{2} d t \cdot \sum_{i=0}^{m}\left\langle n_{i}\right\rangle \cdot\left\langle q_{i}^{2}\right\rangle
$$

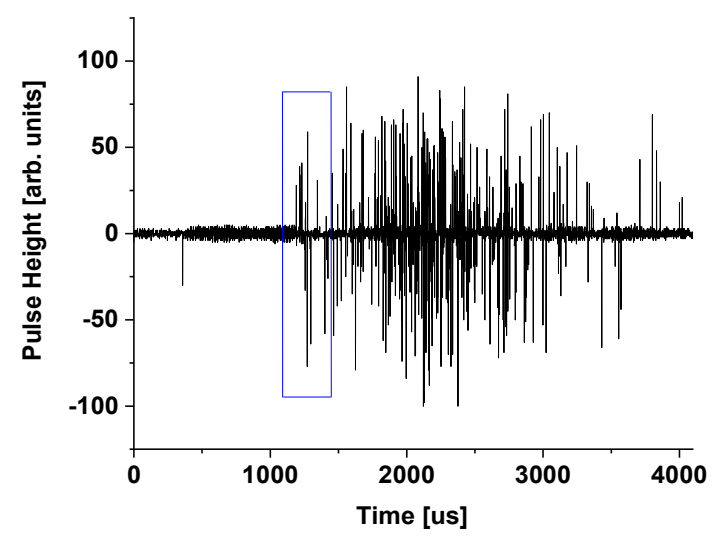

Fig. 3. Resulted waveform after second waveform was subtracted from the first one.

To investigate the Campbell theorem experimentally we divided whole measurement consisting of more than 160000 of reactor bursts into the pieces of 12000 bursts. For each reactor burst we measured the number of detected neutrons, which was fluctuating from burst to burst making distribution presented in Fig.5. There were main group of bursts with the average neutron number $\mathrm{n}_{\mathrm{m}}=168$ per

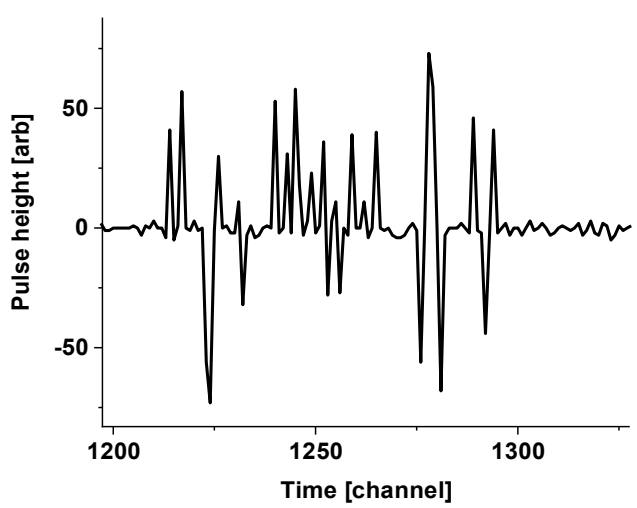

Fig. 4.:The enlarged area from the Fig. 3 to make individual pulses visible

burst and the FWHM=23.9 and a small group with $\mathrm{n}_{1}=131.7$ and FWHM=21.3. Two group caused by the effect of the threshold in peak search algorithm implemented for FC pulse counting. The neutrons were calculated according to number of peaks above the threshold using constant fraction time marking algorithm, described in our previous publications [3]. From the data presented in Fig. 4 one can estimate the time intervals 


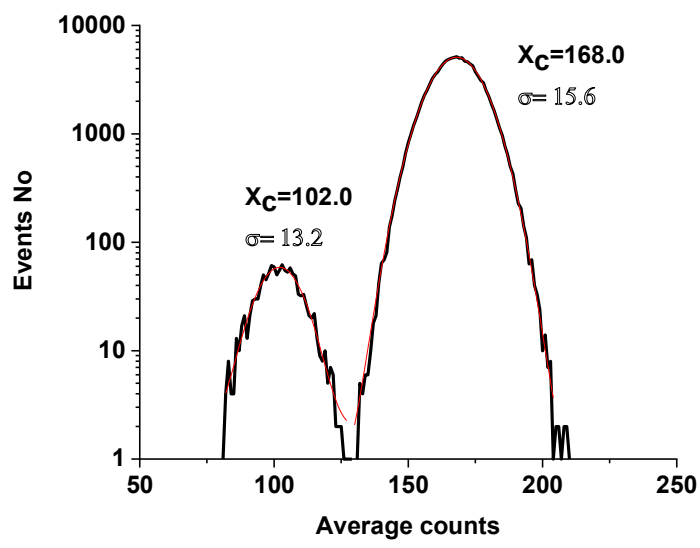

Fig. 5. Distribution of a number of neutrons detected in the set of 200 bursts .

between the sequential pulses presented in Fig. 6 which demonstrates the numbers of events with time difference between successive pulses in the range 15us up to 1250 us. This distribution demonstrates variable counting rate of Poisson distribution inherent to pulsed sources. One of the important features available with measuring technique using DPP is simulating the high counting rates from the set of measurements made at relatively low counting rate.

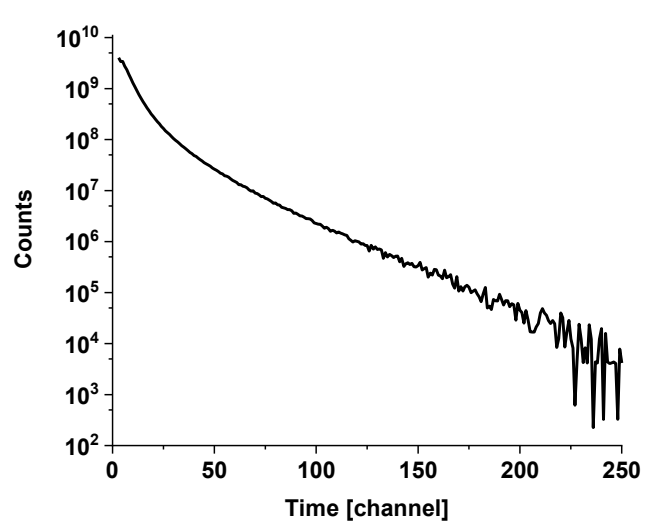

Fig. 6. Distribution of time intervals between successive pulses

In current measurement the full set of data was divided into the smaller subsets, containing 20000 burst each. Taking into account the number of pulses in one burst 168 the number of pulses in $1.6^{*} 10^{5}$ burst would be $\sim 3.0^{*} 10^{7}$. Summing whole pulses into single burst produce single burst, containing $3.0^{*} 10^{7}$ pulses in 20 msec. The most of the pulses overlapped simulating the situation of practically continuous current flow trough detector as demonstrated in Fig. 7. Such situation is exactly the same as it would be with a detector exposed to the neutron flux producing intensity of a detector $1.5 * 10^{9}$ pulses/sec. Using this approach we were able to simulate comparison of the pulsed, the current and the Campbelling techniques with the same data set. From the average pulse count in the single burst we managed estimate the total number of pulses in the acquired data set. To simulate the current mode measurement we summed 160000 successive reactor bursts into single distribution as demonstrated on Fig. 8. Fig.9 demonstrates the share between partially moderated neutrons and gamma radiation plus part of the fast neutrons passed the moderator without interaction from plutonium's fission in the reactor core, used to evaluate the FC relative sensitivity to the prompt neutrons and gammas. Fast prompt fission neutrons produced pulses in the chamber mainly by inducing the fission with cross section about $2 \mathrm{~b}$, whereas prompt fission gammas produce pulses in the FC by directly ionizing the FC's working gas atoms. If the target containing about $6 * 10^{18}$ uranium atoms (double sided $0.5 \mathrm{mg} / \mathrm{cm}^{2}$ ) irradiated by the fast neutron flux $10^{12} \mathrm{n} / \mathrm{cm}^{2}$ would produce

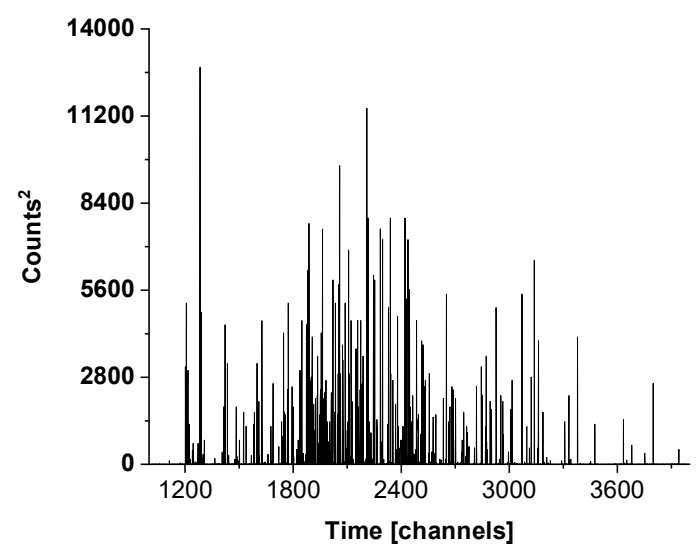

Fig. 7. Autocorrelation function calculated after subtraction of two successive reactor bursts

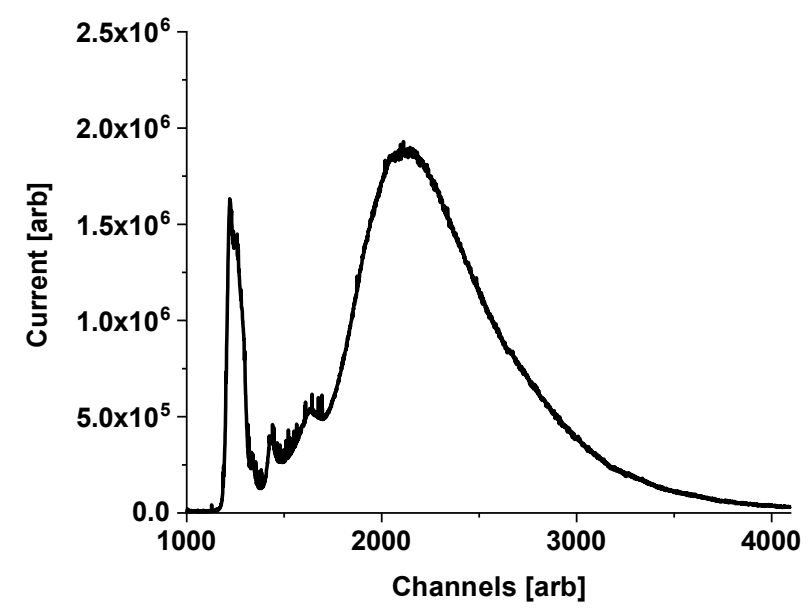

Fig. 8. FC pulses summed for 160000 reactor bursts.

$2 * 10^{6}$ pulse $/ \mathrm{cm}^{2}$ in the FC. According to the plot in Fig. 9 prompt fission gammas plus neutrons escaped the moderator produce by factor 31.08 pulses less. For the thermal neutron region the neutron capture cross section about 250 times higher, meaning that the neutrons would produce by factor 250 more pulses than gammas and the intensity of pulses from gammas would be at least 31.08 times less. From these rough speculation the sensitivity of FC to gamma radiation would be $\sim 7000$ less than sensitivity to thermal neutrons. 


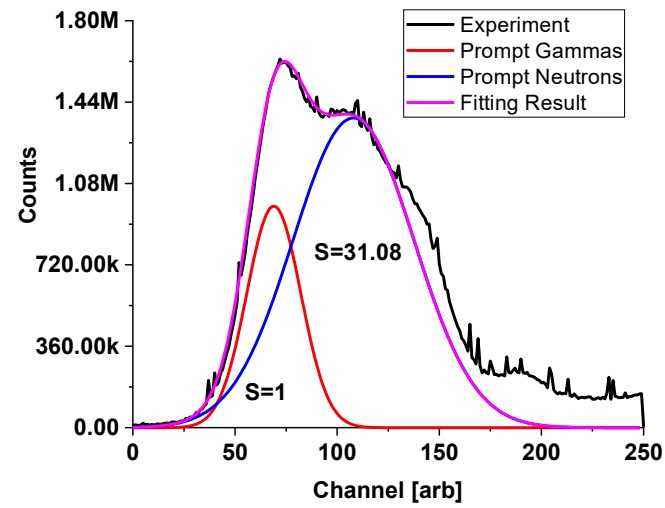

Fig. 9. Radiation from the reactor burst with decomposition into the neutron (partially moderated/resonance) and gamma plus fast neutrons, escaped the moderator, represented by Gaussians. S indicates relative area under the Gaussians.

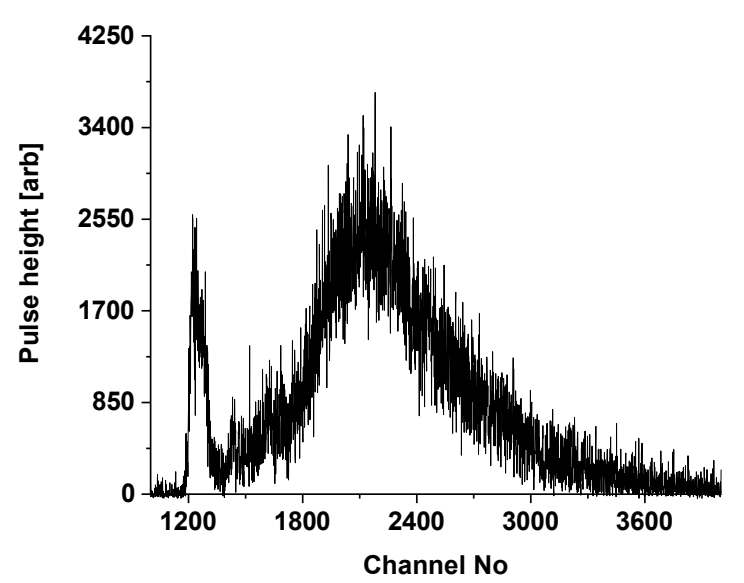

Fig. 10. Group waveform composed of 100 sequential waveforms;

Finally in Fig.11 we have presented results of the current mode and the Campbelling techniques of the pulse rate measurement using FC. Each point was obtained by summing the pulses from the sequential reactor bursts incrementally: the second group burst pulses were added to first one, the third group of burst pulses were added to the second one and so on. Each group consisted of waveforms

acquired in 100 sequential bursts. For the analysis the waveforms were summed creating almost continuous distribution as shown on the graph of the Fig. 10. Then for the current mode measurement the components of the distribution from Fig.10 was integrated producing the number proportional to the pulse intensity in the given group. For the Campbelling technique the components of distribution was squared before integration then the square root of integral was considered as measure of the flux intensity for the given group. Results for the current and Campbelling technique were plotted on the left of Fig. 11 both demonstrating very good linearity.

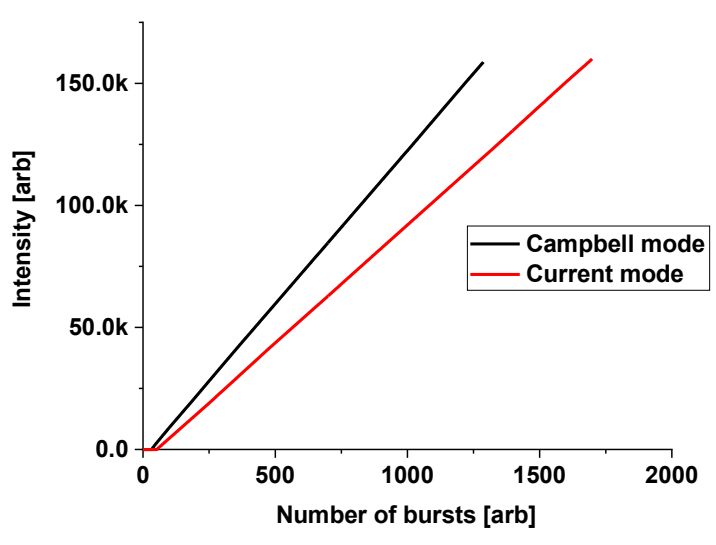

Fig. 11. Comparison of intensity measurement in Campbell and current modes demonstrates very good linearity

\section{Conclusions}

In this paper we report results of out-of-core thermal neutron flux measurement on pulsed nuclear reactor IBR2 using the counting, the current and Campbelling techniques. Counting mode was used to make direct link between the number of detected neutrons and the current flowing through the FC. Pulsed neutron source provided unique possibility to achieve a variable neutron flux density in the same data set. The neutron induced signals result in pulses with much greater charges than pulses from gammas, the mean square signal will weigh the neutron component by the square of the ratio of neutron signal neutron- to gamma ray induced signal [2]. In our work we find this ratio not less than 7000 from rough estimate of experimental data.

1. N.R. Campbell, V.J. Francis, "Theory of Valve and Circuit Noise, JIEE, Vol. 93. Part III, 1946; DOI:10/1049/ji-3-2.1946.0009.

2. G.F. Knoll, Radiation detection and measurements, (Wiley, New York, 2000), p. 522, Chapter 14, IV, Reactor Instrumentation).

3. L. Svetov, Sidorova O., Sh. Zeinalov. In proc. of XXIV International Seminar on Interaction of Neutrons with Nuclei, Dubna, May 24-27, 2016, JINR E3-2017-8, p. 430. 